\title{
Pill camera
}

A 67-year-old man with a history of renal insufficiency due to membranous glomerulonephritis was referred to our endoscopy suite for capsule endoscopy for further evaluation of anemia and recurring positive guaiac fecal occult blood testing. Colonoscopy 8 months earlier and a recent upper gastrointestinal endoscopy had shown no abnormalities. After obtaining informed consent and bowel preparation, wireless capsule endoscopy was done for obscure gastrointestinal bleeding using the PillCam SB video capsule (Given Imaging, Yoqneam, Israel). The capsule study showed rapid small-bowel transit (24 minutes) and angiodysplasias in the right hemicolon. In addition, to our surprise and in contrast to the previously negative colonoscopy, we also found a polypoid lesion in the right hemicolon measuring about $1 \mathrm{~cm}$ in diameter ( $\bullet$ Fig. 1). The patient was scheduled for complete colonoscopy with argon plasma coagulation and polypectomy the following day. Argon plasma coagulation of the angiodysplasias in the cecum and sigmoid colon was carried out successfully. In the ascending colon we found a flesh-colored pill, which had been mistaken for an adenomatous polyp at capsule endoscopy ( Fig. 2). The mucus surrounding the tablet was easily washed off through the colonoscope. Fig. 3 shows a multivitamin tablet with a similar appearance.

The ability of wireless capsule endoscopy to find small-bowel lesions in patients with obscure gastrointestinal bleeding has been demonstrated in a number of studies. Moreover, wireless capsule endoscopy conducted for the detection of small-bowel pathology can occasionally reveal colonic abnormalities missed by previous standard optical endoscopic examinations. Recently, a novel video capsule for capsule endoscopy of the colon has been presented. However, the sensitivity and specificity of the capsule for the detection of colorectal polyps and cancer are still inferior to those of standard colonoscopy. Even more concerning is that in a large multicenter study the colon capsule failed to detect five of the 19 cancers identified by colonoscopy [1]. Moreover, as seen in our case, Gay et al. found false-positive results in $11 \%$ of their sample when using the colon capsule. In contrast to our patient, in whom a large polyp was suspected, these cases mainly involved images that mimicked small polyps $(<6 \mathrm{~mm})$ on colon capsule endoscopy recordings [2]. Finally, regarding the previous negative colonoscopy in our case, it has to be kept in mind that large polyps are rarely missed during lower gastrointestinal endoscopy (with a miss rate of about $2 \%$ for adenomas $\geq 10 \mathrm{~mm}$ as determined by tandem colonoscopy [3]).

\section{Competing interests: None}

Endoscopy_UCTN_Code_CCL_1AC_2AH

\section{S. von Delius, R. M. Schmid, A. Meining} Second Medical Department, Klinikum rechts der Isar der Technischen Universität München, Munich, Germany

\section{References}

1 Van Gossum A, Munoz-Navas M, FernandezUrien I et al. Capsule endoscopy versus colonoscopy for the detection of polyps and cancer. N Engl J Med 2009; 361: 264-270

2 Gay G, Delvaux M, Frederic M, Fassler I. Could the colonic capsule PillCam Colon be clinically useful for selecting patients who deserve a complete colonoscopy? Results of clinical comparison with colonoscopy in the perspective of colorectal cancer screening. Am J Gastroenterol 2009; [Epub ahead of print]

3 van Rijn JC, Reitsma JB, Stoker J et al. Polyp miss rate determined by tandem colonoscopy: a systematic review. Am J Gastroenterol 2006; 101: $343-350$

\section{Bibliography}

DOI $10.1055 / \mathrm{s}-0029-1244143$

Endoscopy 2010; 42: E161

(c) Georg Thieme Verlag KG Stuttgart · New York . ISSN 0013-726X

\section{Corresponding author}

\section{S. von Delius, MD}

Technische Universität München

Klinikum rechts der Isar

Second Medical Department

Ismaninger Str. 22

81675 Munich

Fax: +49-89-41404905

stefan_ruckert@yahoo.de

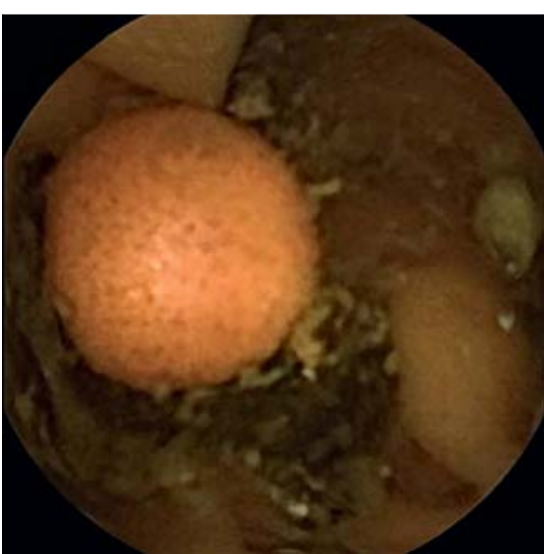

Fig. 1 Video capsule endoscopy showing a polypoid lesion in the right hemicolon.

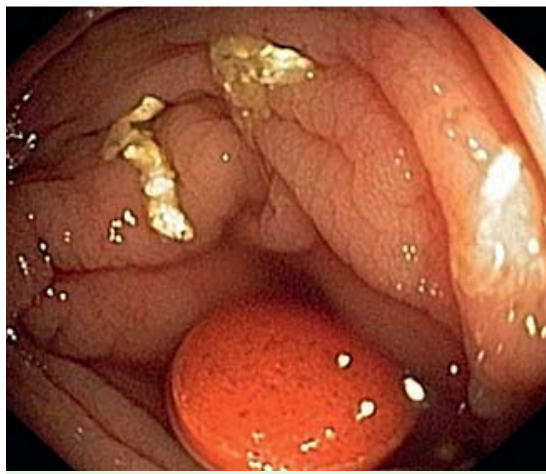

Fig. 2 Further examination revealed the lesion to be a pill.

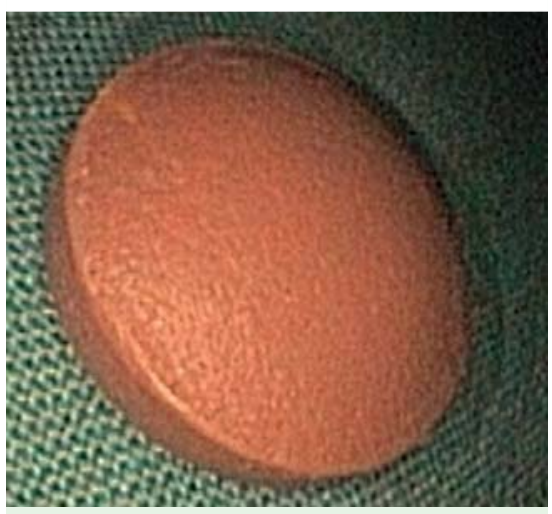

Fig. 3 Similar appearing multivitamin tablet. 